\title{
Philosophiques
}

\section{Temps, durée et mort chez Spinoza}

\section{Bruce Baugh}

Volume 29, numéro 1, printemps 2002

Spinoza sous le prisme de son anthropologie

URI : https://id.erudit.org/iderudit/009561ar

DOI : https://doi.org/10.7202/009561ar

Aller au sommaire du numéro

Éditeur(s)

Société de philosophie du Québec

ISSN

0316-2923 (imprimé)

1492-1391 (numérique)

Découvrir la revue

Citer cet article

Baugh, B. (2002). Temps, durée et mort chez Spinoza. Philosophiques, 29(1),

23-39. https://doi.org/10.7202/009561ar d'utilisation que vous pouvez consulter en ligne.

https://apropos.erudit.org/fr/usagers/politique-dutilisation/ 


\title{
Temps, durée et mort chez Spinoza
}

\author{
BRUCE BAUGH \\ University College of the Cariboo \\ bbaugh@cariboo.bc.ca
}

\begin{abstract}
RÉSUMÉ. - L'Éthique de Spinoza, contrairement aux apparences, présente une riche philosophie du temps, dans laquelle Spinoza distingue entre le temps comme chronologie d'événements datables, ordonnés par la série passé-présent-futur qui est un produit de l'imagination, et le temps comme durée, qui est la «puissance de l'existence » des modes finis existants, et qui est pleinement réel. Quoique Jonathan Bennett soutienne clairement la distinction entre « temps » et «durée », la nature de la durée est plus clairement expliquée par Gilles Deleuze. Selon Deleuze, la « durée » est la «puissance d'exister » des modes finis qui se mesure par les « degrés de puissance » correspondant aux essences modales, plutôt que par la longueur de temps où un mode existe. Cet article utilise l'interprétation de Bennett et de Deleuze pour déterminer à quel point le temps est inclus dans les relations éternelles entre les essences modales, et dans les relations de mouvement et de repos qui constituent ces essences éternelles.
\end{abstract}

\begin{abstract}
Spinoza's Ethics, contrary to appearances, presents a rich philosophy of time, in which Spinoza distinguishes between time as a chronology of dateable events ordered by the series past-present-future, which is a product of the imagination, and time as duration, which is the "power of existence" of actual finite modes, and is fully real. The distinction between "time" and "duration" is clearly argued for by Jonathan Bennett. The nature of "duration," however, is more clearly explained by Gilles Deleuze. According to Deleuze, "duration" is a "power of existing" of finite modes that is measured by "degrees of power" corresponding to modal essences, rather than by the length of time a mode exists. This paper concludes by using Bennett's and Deleuze's interpretations to determine the extent to which time is implicated in the eternal relations among modal essences, and in the relations of motion and rest constitutive of those eternal essences.
\end{abstract}

L'Éthique ${ }^{1}$ de Spinoza peut-elle nous donner une philosophie du temps ? À première vue, l'idée semble absurde. Spinoza est le philosophe qui a le plus insisté sur le fait que toute vérité authentique devait être saisie sub specie aeternitatis, du point de vue de l'éternité, et que l'éternité elle-même paraissait entièrement atemporelle: " L'éternité ne peut se définir par le temps ni avoir aucune relation au temps » (E V P23 Scolie), "l'éternité ne peut s'expliquer par la durée » (E V P29 Démonstration), « dans l'éternité il n'y a d'ailleurs ni quand, ni avant, ni après » (E I P33 Scolie 2). Il s'ensuit que lorsque la raison perçoit

1. Spinoza, Euvres, 4 vol. éd. Ch. Appuhn, Paris, GF, 1965, vol. III Éthique. Pour les références à l'Éthique $(E)$, les parties sont désignées par des chiffres romains et les propositions par P suivi du numéro de la proposition. Par exemple, E I P18 désigne la proposition 18 de la première partie. 
les choses du point de vue de l'éternité (sub quadam specie aeternitatis), elle les conçoit « sans aucune relation au temps » (E II P44 Corollaire 2 Démonstration). Le temps, d'un autre côté, semblerait être illusoire et source d'illusions : " Nul ne doute d'ailleurs que nous n'imaginions [...] le temps » (E II P44 Corollaire 1 Scolie). Il ne faut pas s'étonner alors que l'on ait accusé Spinoza de ne pas «prendre au sérieux le temps $»^{2}$. Dans certaines interprétations, même, on dirait presque que Spinoza se passe complètement du temps au profit d' " une nécessité logique atemporelle », remplaçant la séquence temporelle des événements arrangés selon des relations $d^{\prime}$ '" avant » et d' "après " par " une séquence logique de modifications nécessairement reliées les unes aux autres " par des relations d'implication logique. Dans un tel système, "les déterminations de temps n'ont pas de place, tout comme elles n'ont pas de place en géométrie $»^{3}$.

Il y a des éléments textuels venant soutenir cette vision de Spinoza. Pourtant, tout le monde n'est pas d'accord avec elle. Jonathan Bennett, dans son commentaire plutôt idiosyncrasique de l'Éthique, prétend que "Spinoza attribue la durée à Dieu ou la Nature - un point qui n'aurait jamais d̂̀ être sujet à controverse ${ }^{4}$. Si Bennett a raison, alors Spinoza doit concevoir la durée comme entièrement réelle, aussi réelle que Dieu ou la substance. Partant d'une perspective philosophique très différente, Gilles Deleuze trouve dans la manière dont Spinoza parle de la durée une théorie du temps dans laquelle le commencement, ou le fait de venir à l'existence, est essentiel, mais pas la fin ${ }^{5}$. La mort est un accident, bien qu'inévitable pour les « modes finis ", et le but de la vie devrait être de vivre "si intensément [...] que la mort, (...) toujours extérieure, soit peu de chose " pour nous (SPP p. 59). Pour rendre compte de cela, il nous faut expliquer le rapport de la mort à la durée, et tenter de fournir une explication du statut ontologique de la durée elle-même. Bennett et Deleuze sont tous les deux d'accord pour affirmer que la durée est réelle, et que les interprétations de l'éternité comme un cadre de rapports purement logiques hors du temps sont «idéalistes » (Deleuze) et «incorrectes » (Bennett). Étant donné cet accord surprenant entre des philosophes d'orientations aussi divergentes qu'eux deux, il peut être bon d'envisager à nouveau la question du temps, de la durée et de l'éternité chez Spinoza. Dans ce qui suit, nous regarderons tout d'abord de plus près ce que dit l'Éthique sur cette question, puis nous examinerons l'interprétation proposée par Deleuze de la durée et de

2. Stuart Hampshire attribue cette accusation à Samuel Alexander en se rangeant à son avis. Voir Hampshire, Stuart, Spinoza, Harmondsworth, Penguin, 1978, p. 196.

3. Hampshire, Spinoza, p. 174, 195.

4. "Spinoza attributes duration to God or Nature-a point which should never have been controversial ", Bennett, Jonathan, A Study of Spinoza's Ethics, Indianapolis, Hackett, 1984, p. 207. Nos italiques.

5. Deleuze, Gilles, Spinoza et le problème de l'expression, Paris, Éditions de Minuit, 1968, désormais SPE; et Spinoza : Philosophie pratique, Paris, Éditions de Minuit, 1981, désormais $S P P$. 
la mort chez Spinoza. Enfin, nous envisagerons les implications de cette interprétation pour une théorie spinoziste du temps.

\section{Le temps et l'imagination}

Les commentateurs qui attribuent à Spinoza l'idée que le temps et la durée sont aussi "irréels » ou produits par l'imagination l'un que l'autre ont tendance à concevoir la durée et le temps comme plus ou moins équivalents, et certainement comme ayant le même statut ontologique. Bennett s'oppose à cette idée en soutenant que Spinoza définit le temps et la durée différemment ; que pour Spinoza, le temps n'est pas fondamentalement réel, mais que la durée l'est ${ }^{6}$. Si on suit l'indication donnée par Bennett, on peut trouver trois usages du terme "temps " : le temps comme mesure, ou comme une longueur déterminée de temps; le temps comme relations temporelles d'avant et d'après, ou de passé, présent et futur ; et les moments précis dans le temps, c'est-à-dire le «moment où » quelque chose se produit. Toutes ces conceptions, dit Spinoza, sont des produits de notre imagination.

Comment mesure-t-on un laps de temps ? Par une comparaison des impressions sensorielles (images) que nous avons du mouvement de deux corps : "nous imaginons des corps se mouvant les uns plus lentement ou plus vite que les autres, ou avec une vitesse égale » (E II P44 Corollaire 1 Scolie). Le mouvement ou la vitesse d'un corps mesure ceux d'un autre : le mouvement des nombres sur une horloge mesure le temps qu'il a fallu au coureur pour parcourir cent mètres, le mouvement du soleil mesure quelle portion du jour il faut pour nourrir le bétail, etc. Le mouvement de certains corps nous permet aussi de dire « quand » quelque chose est arrivé : il y a dix secondes, à midi, au milieu de la nuit, lors de la dernière pleine lune. Il apparaît clairement que toutes ces mesures sont conventionnelles plutôt qu'elles ne sont enracinées dans la nature profonde des choses : c'est par commodité que nous mesurons et datons le temps par le mouvement des horloges, la rotation de la Terre sur son axe, l'orbite de la Lune. Tous les mouvements que l'on utilise pour mesurer le temps peuvent être mesurés par d'autres mouvements, aucun de ceux-ci n'étant " fondamental ", mais tous étant relatifs aux autres mouvements qui les mesurent ou les datent. Pourtant, tous les mouvements qu'on utilise pour mesurer le temps ont une certaine régularité dans l'expérience qui nous permet d'anticiper le futur et de nous souvenir du passé, à partir d'associations d'idées. Ainsi, pour reprendre l'exemple de Spinoza, si je vois Pierre le matin, Paul le midi, et Siméon le soir et que cela se produit avec une certaine régularité, j'associerai alors Pierre avec la position du soleil le matin, Paul avec celle du midi, et Siméon avec sa position le soir (voir E II P44 Scolie), de telle sorte que lorsque je verrai le soleil dans une certaine posi-

6. Bennett, A Study of Spinoza's Ethics, p. 196-211. 
tion (quand je le verrai à une certaine « heure de la journée »), je serai amené à penser à Pierre, Paul ou Siméon, et à attendre, anticiper ou " me souvenir " de leur présence : s'il est midi, Paul devrait être là, Siméon sera là plus tard, et Pierre doit être déjà venu. Mon sentiment concernant le «quand » et le « combien de temps » est donc fondé sur une expérience personnelle relativement fortuite, et plus cette expérience d'une conjonction d'événements et de mouvements qui les mesurent et les datent est variable et irrégulière, moins j'ai de certitudes à propos des événements présents, passés et futurs.

On mesure donc un laps de temps en comparant deux mouvements, un qui fonctionne comme une mesure, l'autre qui représente l'événement mesuré : on ne peut répondre à la question de savoir " quand " un événement se produit qu'en recourant à une mesure temporelle (il y a deux jours, pendant les cinq dernières minutes, dans un mois). En fait, nous mesurons et datons un événement à travers un autre qui n'a pas de relation nécessaire avec, mais qui se trouve par coïncidence se produire " en même temps ", et qui est du type à se produire avec une certaine régularité dans le champ de notre expérience. Cette régularité est celle d'un " cours des événements ", de la séquence selon laquelle les choses se suivent les unes les autres (comme la séquence des points ou nombres par lesquels passe successivement l'aiguille d'une horloge). Cependant, le caractère non fiable et incertain des régularités de l'expérience, de quelque sorte que ce soit, provient de leur manque de nécessité : ce sont des sortes d'experientiae vagae, d'expériences et de perceptions qui se suivent " sans ordre pour l'entendement " (E II P40 Scolie 2) ou nécessité logique, mais qui, bien plutôt, proviennent de la manière fortuite dont les circonstances externes agissent sur le corps et l'esprit (E II P29 Scolie). Au lieu de percevoir des relations logiques d'accord ou de désaccord, de différence ou de similitude, de contradiction ou d'implication, l'esprit n'enregistre qu'une succession d'états arrangés en termes d' " avant » et d' "après ". Lorsqu'un événement en précède régulièrement un autre, on croit qu'il est la " cause » de l'événement qui suit, tombant alors presque naturellement dans le piège du sophisme post hoc ergo propter hoc. Mais quand on utilise la raison pour " considérer plusieurs choses à la fois " plutôt que de manière successive, les rapports d'implication logique supplantent les rapports temporels de succession, et les idées d' " avant » et d' "après " sont remplacées par celles de "raison suffisante " (cause) et de "conclusion" (effet). Les rapports temporels d'avant et d'après, comme les mesures temporelles et les dates, ne peuvent jamais faire partie de la connaissance véritable, aussi fermement enracinées "dans l'ordre commun de la nature " soient-elles, ou dans les habitudes d'association basées sur l'expérience ordinaire (voir E II P29 Corollaire).

Spinoza rend compte de manière identique de la distance temporelle dans la mémoire et l'anticipation. On pourrait penser qu'un événement est plus ou moins lointain dans le passé ou dans le futur selon la quantité de temps qui se trouve entre le présent et le passé ou le futur, sauf que le " temps » n'est 
pas une entité ou une quantité réelle mais une fiction issue de l'imagination (voir E IV Définition 6, E IV P9 avec Scolie et Corollaire, et E IV P10). Dans l'expérience, la distance temporelle est reflétée par le degré d'intensité d'une image : plus la chose est " proche » dans le temps, et plus j'en suis affecté intensément. Spinoza explique que l'intensité est fonction de la non-interférence d'autres images ou affections du corps : " nous appellerons images des choses les affections du corps humain dont les idées nous représentent les choses extérieures comme nous étant présentes » (E II P17 Scolie) ; et l'esprit tiendra une chose pour présente ou pour cause opérante effective de son idée en nous " aussi longtemps que nous n'imagin[er]ons rien qui exclue l'existence présente de la cause extérieure »( $E$ IV P9 Démonstration). Le problème est de savoir comment comprendre cette "exclusion ". Bennett suggère une " incompatibilité logique ${ }^{7}$, mais cela nous apparaît clairement improbable, étant donné que Spinoza décrit ici les illusions de l'expérience imaginative d'une manière que n'importe quelle analyse logique écarterait. Bien plutôt, une fois encore, la "durée de temps " se mesure par des images et des expériences associées, et ce qui " exclut » l'existence présente d'une chose, c'est une série d'images interposées entre l'image temporellement " distante » et les images présentes de manière plus immédiate. Par exemple, j’appelle à mon esprit une image, ou j' "imagine " maintenant, à midi, que j'ai arrêté mon réveil à sept heures du matin, mais les images de toutes les choses qui se sont passées entre sept heures et maintenant m'empêchent de concevoir l'image que j'ai de mon réveil arrêté à sept heures comme une chose présente ; je dois reléguer cet événement et cette image dans le passé, la distance entre le présent et le passé étant mesurée par les images qui interviennent et qui sont temporellement ordonnées en "avant " et " après " par experientia vaga. Voilà ce que c'est que "se souvenir » d'un événement passé : c'est en avoir une image qui est représentée comme appartenant au "passé » (voir E III P18 Démonstration et Scolie 1). De la même manière, anticiper un événement futur revient à se le représenter comme appartenant au futur, et donc pas au présent, sa présence étant exclue par les images de tous les événements interposés entre maintenant et le futur. Par exemple, en anticipant mon arrivée là où je veux me rendre, mon esprit passe en revue tous les endroits par lesquels je devrai passer pour y arriver. C'est cette interposition d'autres images représentant les états successifs du monde comme ils apparaissent dans l'expérience sensible qui « exclut " la présence d'une chose et rend son image moins intense : plus il y a d'interférence en provenance d'autres images, moins il y a d'intensité et plus on imagine de distance temporelle.

Ces réflexions sur le traitement que fait Spinoza du temps nous permettent de tirer quelques conclusions préliminaires. Premièrement, on voit maintenant avec évidence comment le temps est un produit de l'imagination en ce qui concerne l'écoulement du temps, l'ordre temporel (avant/après) et la

7. Ibid., p. 200-202. 
« distance » entre le présent et le passé ou le futur. Deuxièmement, on voit que l'élément premier de ce temps phénoménal chronologique est la mesure qui provient de la comparaison d'un mouvement ou d'un événement avec un autre, et que cette mesure suppose une séquence d'images qui suivent « l'ordre commun de la nature ", c'est-à-dire la chaîne fortuite d'effets du milieu sur le corps et l'esprit. Le caractère arbitraire d'une telle mesure est évident par soimême : quand on utilise son imagination pour déterminer la quantité ou le temps, en considérant les deux comme séparés de la substance infinie, " proviennent le temps et la mesure. Le temps sert à délimiter la durée, la mesure, à délimiter la grandeur ", ce qui nous permet de " fixer à volonté [...] l'existence et la durée [des modes], la concevoir plus grande ou plus petite et la diviser en parties » (Lettre 12).

\section{Durée}

Le temps, fondé sur l'imagination, est la mesure de la durée, mais il n'est pas la durée. La durée authentique est reliée aux " choses éternelles ", à Dieu ou la Nature comme causes de toutes choses (Lettre 12), et elle appartient à l'existence des modes en tant qu'ils sont déterminés par " une certaine nécessité éternelle " appartenant à la puissance d'agir de Dieu (voir E V P22, E I P16), plutôt qu'elle n'est reliée à la manière dont les modes nous apparaissent à travers l'imagination. Nier la réalité de la durée reviendrait à nier la réalité de l'existence des modes, ce qui est absurde. En quel sens, cependant, la durée est-elle réelle?

Spinoza indique dans la lettre 12 que la durée est une, infinie et indivisible, ce de quoi Bennett conclut déjà que la durée infinie est une durée d'une longueur infinie, équivalente à la sempiternité ou à une durée sans début ni fin $^{8}$. C'est toutefois là aller trop vite en conclusions. Une durée d'une longueur quelconque, même d'une longueur infinie, serait encore une durée conçue par l'imagination et par la mesure. L'infinité de la durée, comme celle de la substance, n'est pas mesurable (E I P15 Scolie). De plus, Spinoza n'attribuerait pas de réalité supérieure à une chose parce qu'elle persisterait plus dans le temps ; au contraire, la réalité d'une chose est « l'essence d'une chose quelconque en tant qu'elle existe et produit quelque effet en une certaine manière, n'ayant nul égard à sa durée ", c'est-à-dire sans référence à une durée de temps fixe et déterminée, même d'une extension infinie ( $E$ IV Préface). Et c'est sûrement vrai : nous ne dirions pas que Bertrand Russell était "plus vrai » qu'Albert Camus simplement parce qu'il a vécu beaucoup plus longtemps. On doit rendre compte de la réalité et de l'" infinité » de la durée d'une manière différente.

8. Ibid., p. 204-205. 
Si l'on regarde les passages où Spinoza parle de la durée, on s'aperçoit qu'il utilise ce terme en deux sens, l'un étant celui d'un laps de temps fixe (comme dans la préface de la quatrième partie), l'autre ne comportant aucune référence essentielle à la mesure temporelle, ou même étant opposé à la mesure temporelle (comme dans la Lettre 12). Il y a alors la durée mesurée par le temps, et la durée en elle-même, qui n'est ni une mesure ni quelque chose de mesuré. Ainsi, quand Spinoza dit que " nous n'attribuons à l'âme humaine aucune durée pouvant se définir par le temps, sinon en tant qu'elle exprime l'existence actuelle du Corps, laquelle s'explique par la durée et peut se [limiter] par le temps " (E V P23 Démonstration) ${ }^{9}$, il oppose la durée ellemême, qui est l'existence actuelle d'une chose, à la durée telle qu'elle est mesurée par le temps.

En elle-même, la durée, ou l'existence actuelle, n'a aucune limite ni mesure (E II P45 Démonstration). La limitation est extrinsèque et non intrinsèque ; elle provient non de l'existence de la chose, mais d'une comparaison entre celle-ci et quelque chose d'autre : « la perfection et l'imperfection sont en réalité des modes de penser ", car " pour autant qu'on attribue [aux phénomènes naturels] quelque chose impliquant une négation, telle qu'une limite, une fin, une impuissance etc. » (E IV Préface), et que nous les considérons comme imparfaits, nous comparons la manière dont ils affectent nos esprits avec la manière dont nos esprits sont affectés par quelque chose d'autre, au lieu de les envisager en rapport avec leur propre essence. Comme le dit Deleuze, « tout est perfection dans la nature [...]. Toutes les formes d'être s'affirment sans limitation, donc s'attribuent à quelque chose d'absolu » (SPE p. 71 ; voir E IV Préface), c'est-à-dire à la substance, qui est conçue par elle-même ( $E$ I Définition 3 ), et est pour cette raison infinie ( $E$ I P8). Par conséquent, tant qu'il existe, "le mode [...] n'a d'autre puissance qu'actuelle : à chaque instant il est tout ce qu'il peut être, sa puissance est son essence » (SPE p. 83 ; voir SPP p. 58, 124-125). Pour ce qu'il en est de la durée, si elle n'est pas mesurée ou datée en référence à quelque chose d'autre mais conçue comme une existence actuelle expliquée par l'essence de la chose, alors la durée est conçue sub specie aeternitatis, et expliquée "avec une sorte de nécessité éternelle " par l'essence de Dieu $(E \mathrm{~V}$ P22, P29 Scolie), et c'est dans cette mesure qu'une chose est réellement (E V P30 Démonstration). En d'autres mots, l'existence actuelle ou la durée d'une chose, considérée comme le résultat nécessaire de sa cause efficiente et de lois éternelles de la nature, est une force d'existence, une vis existendi (E II P45 Scolie) exprimant la nécessité de sa nature ou de son essence - une essence qui détermine la manière précise dont la chose agit et existe, mais qui n'est en aucun cas définie par une limite temporelle ou des moments datables (le passé, le présent, le futur,

9. Nous nous séparons d'Appuhn en français et de Shirley en anglais qui écrivent ici "définir " et nous suivons White dans son choix de "limiter ", pour des raisons qui vont devenir apparentes. Voir Wild, John, ed., Spinoza : Selections, New York, Charles Scribner's Sons, 1958 , p. 384. 
voir E IV Préface). Dans la mesure où l'existence d'une chose « suit de la nécessité de la nature de Dieu » (sa cause efficiente), elle ne peut être différente et elle participe de la nécessité éternelle de « la nature de l'existence elle-même » (E II P45 Démonstration et Scolie, E I Définition 8), c'est-à-dire qu'elle participe de Dieu, conçu en tant que cause efficiente non seulement de la venue à l'existence d'une chose, mais aussi de son essence (E I P25) et de sa continuation dans l'existence (E I P24 Corollaire). C'est là, bien plus qu'une durée quelconque de temps, ce qui rend les choses « réelles » et définit leur durée ou leur existence en tant que modes (voir Lettre 12).

Ceci explique la définition que donne Spinoza de la durée comme " une continuation indéfinie de l'existence ", puisqu'aucune limite à cette existence ne suit de l'essence de la chose, qui détermine simplement comment (et non combien de temps) une chose existe et agit, et puisqu'elle ne suit pas plus de la cause efficiente de cette chose, "laquelle [...] pose nécessairement l'existence de la chose, mais ne l'ôte pas ", la cause efficiente étant seulement à l'origine de la venue à l'existence de la chose ( $E$ II Définition 5 et Explication). Une fois qu'une chose a commencé d'exister, alors elle " pourra persévérer toujours dans l'existence avec la même force par quoi elle a commencé d'exister » (E IV Préface), cette "force » étant déterminée par son essence (qui exprime la nécessité des lois éternelles de la nature) et sa cause efficiente, les deux " affirmant » ou " posant » son existence ( $E$ III P4, IV Préface, EPS p. 211). Puisque son essence et sa cause efficiente affirment plutôt qu'elles ne nient son existence, « nulle chose ne peut être détruite sinon par une cause extérieure " (E III P4), et "si elle n'est détruite par aucune cause extérieure, elle continuera d'exister " (E III P8 Démonstration). Aucune limitation ne suit de l'essence ou de la cause efficiente d'une chose, c'est-à-dire, ne suit de sa réalité, et par conséquent la longueur temporelle de la durée d'une chose "n'enveloppe aucun temps fini mais un temps indéfini » ( $E$ III P8), " puisque l'essence des choses n'enveloppe aucun temps certain et déterminé d'existence » ( $E$ IV Préface). De la même manière qu'un corps simple continuera d'être en mouvement tant qu'un autre corps ne l'aura pas fait s'arrêter ou changer son cours (E II P13 Lemme 3 et Corollaire), de la même manière aussi l'existence, une fois commencée, continuera jusqu'à ce qu'une cause extérieure plus puissante la force à s'arrêter.

En bref, la durée est un vecteur plutôt qu'une ligne d'une longueur déterminée : elle a nécessairement un début, mais elle n'a pas, en elle-même, de fin (SPP p. 86-87). À ce titre, la naissance et la mort sont asymétriques. Puisque toute chose finie s'efforce de "maintenir et affirmer son existence " indéfiniment (SPP p. 135-136), la durée est la « continuation d'existence à partir d'un commencement " (SPP p. 86, 136 ; SPE p. 178-179, 290). Toute fin qui viendrait la limiter ne suivrait pas de sa propre nature ou essence, mais d'une cause extrinsèque, de quelque chose de fortuit et d'accidentel par rapport à sa propre nature. Toute mort est "brutale, violente et fortuite " (SPE p. 218), elle est le résultat de la rencontre d'une chose finie avec « une autre 
plus puissante, par laquelle la première peut être détruite » (E IV Axiome). Et puisqu' ' il n'est donné dans la nature aucune chose singulière qu'il n'en soit donné une autre plus puissante et plus forte » ( $E$ IV Axiome), de telles rencontres sont inévitables, et qui plus est, nécessaires du point de vue de la nature comme tout. Mais cette nécessité est purement extérieure, due "aux lois d'un déterminisme externe ", plutôt qu'elle ne suit de l'essence d'une chose (SPP p. 21, 59-62, 121, 137-138). C'est n'est pas du tout une nécessité logique, comme l'est la nécessaire inexistence de cercles carrés (E I P33 Scolie $1)$, mais bien la mort contingente d'une chose contingente, c'est-à-dire d'une chose dont l'essence ne pose ou n'exclut pas nécessairement son existence $(E$ II Axiome 1, E IV Définition 3). Ainsi, bien que la mort soit inévitable et entièrement nécessaire eu égard à l'ordre total des causes dans la nature, elle est entièrement extrinsèque et contingente par rapport à l'essence d'un mode (SPP p. 59-61, 121 ; SPE p. 228 ; E I P33 Scolie 1). La durée, au contraire, est l'existence même des modes finis, et puisque cette existence ne suit pas nécessairement de leur essence, elle doit être posée par une cause efficiente, l'essence et la cause constituant ensemble un "dynamisme d'après lequel l'essence s'affirme dans l'existence " (SPE p. 213). Toute existence modale requiert d'être posée ou affirmée pour être, mais elle n'a besoin de négation d'aucune sorte. C'est pourquoi, quoique la durée ait une relation essentielle avec la venue à l'être (generatione), elle n'a aucun lien essentiel avec la destruction ou la mort (corruptione) ${ }^{10}$. Au regard de sa propre essence, la durée d'une chose est pleinement positive et n'inclut aucune privation ou limite.

\section{La mort chez Spinoza selon Deleuze}

La section précédente a suivi de très près l'interprétation proposée par Gilles Deleuze de la philosophie de Spinoza comme pleinement positive et affirmative (SPE p. 51), comme " une logique de l'affirmation pure, de la qualité illimitée » (SPE p. 69). Comment, toutefois, cette lecture peut-elle être conciliée avec la finitude des modes et de leur essence ? Si un mode, ou son essence, est infini, alors il participe de l'éternité et de la nécessité de Dieu, et « ne peut avoir une durée déterminée » ( $E$ I P21 Démonstration). Il n'est que trop évident que ce n'est pas le cas : "l'essence de l'homme n'enveloppe pas l'existence nécessaire » ( $E$ II Axiome 1), d'où il suit que la substance ou l'être nécessaire ne constitue pas l'essence ou la forme de l'homme (E II P10). Notre durée ne découle pas de notre essence, mais elle est déterminée par des causes extérieures à nous suivant "l'ordre commun de la nature et la structure de l'univers » (E II P30 Démonstration). À la fois notre essence et notre existence sont finies. La question est de savoir comment cela peut être conçu sans que la négation soit intrinsèque à l'essence ou à l'existence des modes.

10. Contrairement à ce qu'en disent quelques interprétations, comme celle de Hampshire (voir Spinoza, p. 173). 
Pour commencer, la finitude des essences modales ne doit pas, selon Deleuze, être comprise comme une privation d'une perfection plus grande : chaque essence finie est un degré de qualité (SPE p. 166), un degré de puissance ou d'intensité, ou " une puissance d'exister et d'agir » (SPE p. 78-79) qui est totalement positive et "singulière en elle-même " (SPE p. 166, 173, 179-182 ; SPP p. 99, 135). En bref, une essence est un mode d'être qualitativement singulier, une modification déterminée des attributs infinis et éternels de Dieu (SPE p. 192-193). En ce sens, les essences ne sont pas de simples possibilités métaphysiques ou logiques; elles sont actuelles : l'essence d'une chose, ou sa forme, correspond à un rapport (ratio) de mouvement et de repos parmi les corps simples qui la constituent, cette forme étant ce qui fait de cette chose un individu déterminé ( $E$ II P13 Lemmes 1, 4, 5, 6 et 7). Parce que le mouvement et le repos appartiennent à l'étendue et sont physiques, les essences finies correspondant aux rapports de mouvement et de repos sont également physiques, sont des " parties de puissance, c'est-à-dire, (...) degrés de puissance ou d'intensité " physique (SPE p. 173, 291 ; SPP p. 99-100). De plus, quoique les essences finies soient causées par Dieu plutôt que par ellesmêmes ou entre elles (SPE p. 174-175, E I P25), elles sont néanmoins éternelles ; elles expriment la nécessité de la nature éternelle de Dieu et elles "s'accordent» les unes les autres par leur cause commune. Chaque essence, alors, exprime à la fois Dieu et toutes les autres essences dans " un système total, un ensemble actuellement infini » (SPE, p. 177, 283, 290-291). En bref, les essences sont réelles plutôt qu'idéales, actuelles plutôt que possibles, éternelles plutôt que relevant d'une durée temporelle. Chaque essence est, en effet, un système singulier de rapports qui gouverne les rapports entre les parties constituantes d'un mode fini existant, le rapport constituant entre ces parties étant un rapport singulier de mouvement et de repos. Ni les essences, ni les rapports constituants ne contiennent en eux-mêmes de négation ou de privation ; ils sont « limités » seulement au sens comparatif selon lequel une qualité diffère d'une autre, mais l'impossibilité pour une qualité ou une essence d'être une qualité ou une essence autre n'est pas un manque ou une erreur dans sa nature (voir Lettre 21).

Deleuze soutient en fait que tant l'essence d'un mode fini (son degré d'intensité ou de puissance) que son rapport constitutif (la composition de ses parties constituantes selon les lois de la nature) sont éternels (voir SPE, ch. 15). Un système de rapports est indépendant de son actualisation dans des corps existants (SPE p. 230, 257) : il ne manque de rien, " même quand les modes correspondants n'existent pas ou n'existent plus » (SPE p. 209). Chaque rapport constitutif est, de la même manière, "une vérité éternelle " appartenant aux rapports entre les " parties extensives » innombrables d'un corps, aussi indifférente à la question de savoir si les corps simples quelconques sont arrangés selon ce rapport qu'à la question de savoir quels corps simples sont impliqués. C'est seulement le rapport de mouvement et de repos qui est essentiel, pas les corps simples particuliers qui sont en relation (SPE 
p. 214-215) ; par conséquent les essences et les rapports constitutifs ne sont pas affectés par l'existence ou l'inexistence des choses qui leur correspondent (SPE p. 290-291 ; SPP p. 138 ; E V P22 Démonstration).

Seule l'existence actuelle des modes finis a une durée et peut être mesurée par le temps, et cette existence consiste en un très grand nombre de parties extensives qui sont, les unes par rapport aux autres, dans une proportion par laquelle une essence est exprimée (SPE p. 183-184, 191-192, 290 ; SPP p. 124, 134-135). Puisque la rencontre de ces parties extrinsèques et extensives est déterminée par "des lois purement mécaniques ", des " lois de composition et de décomposition des rapports ", " les modes passent à l'existence, et cessent d'exister, en vertu de lois extérieures à leurs essences" (SPE p. 192). Les parties extensives sont par leur nature déterminées de l'extérieur (SPE p. 173) par d'autres parties extensives qu'elles peuvent rencontrer (par les lois de cause à effet), et donc l'effet que les autres parties extensives ont sur un mode est expliqué par un déterminisme externe plutôt que par son seul degré de puissance propre. Ainsi, quoiqu'une essence soit exprimée par des parties extensives dans le rapport approprié, elle n'est pas constituée par ces parties (SPE p. 290, SPP p. 124-125), et n'est donc pas affectée quand les parties entrent dans un autre rapport qui s'avère incompatible avec elle (SPE p. 230). Par suite, "chaque mode dure tant que les parties restent sous le rapport qui le caractérise " (SPE p. 194), et cesse d'être par une "rencontre hasardeuse " dans laquelle ses parties extrinsèques sont déterminées de l'extérieur à entrer dans un rapport qui ne peut pas être combiné avec le sien propre (SPE p. 216217 ; SPP p. 33, 46-48). " Si la mort est inévitable, ce n’est nullement parce qu'elle serait intérieure au mode existant ; c'est au contraire parce que le mode existant est nécessairement ouvert sur le dehors [...], parce qu'il rencontre nécessairement d'autres modes existants capables de léser un de ses rapports vitaux » (SPP p. 137).

La finitude n'est rien d'autre que le fait que « la force avec laquelle un homme persévère dans l'existence [soit] [...] surpassée infiniment par la puissance des causes extérieures » (E IV P3), certaines de celles-ci étant capables de faire en sorte qu' " un autre rapport de mouvement et de repos s'établisse entre elles [les parties du corps humain] » d'une manière qui en détruit la vie (E IV P39 Scolie). Cette limite extérieure ne détermine pas le caractère spécifique du mode ; "la spécificité du mode repose moins sur sa finitude que sur le type d'infini qui lui revient » (SPP p. 119), ou sur la spécificité de son essence ou " degré de puissance ». Il n'y a rien dans l'idée de durée elle-même qui implique une fin.

Pourtant, comment se fait-il que la durée temporelle puisse avoir un commencement de manière nécessaire et une fin de manière "accidentelle " seulement ? À moins que les parties extensives des modes soient continuellement créées et portées à l'existence (une idée que Spinoza rejetterait avec véhémence), la venue à l'existence ou la durée d'une chose sont nécessairement et simultanément la destruction d'autres choses puisqu'elles requièrent que les 
parties extensives qui avaient été subsumées sous d'autres rapports soient subsumées sous un nouveau rapport, incompatible avec l'ancien. La naissance de toute chose est la mort d'une autre ; et si les commencements sont nécessaires, il doit s'ensuivre que les fins le sont aussi. De surcroît, si la mort ou la destruction d'une chose est " extérieure ", dans la mesure où elle suit non pas de l'essence du mode, mais du déterminisme externe qui gouverne ses parties extensives, alors l'existence et la durée du mode sont tout aussi extérieures : l'existence n'ajoute ni ne retranche rien à l'essence d'un mode (voir E II P8), et elle ne suit jamais de cette essence (E I P24, E II P30 Démonstration). Dans ce cas, cependant, une essence éternelle devrait sembler être éternellement morte, n'ayant aucun des mouvements et variations de la vie. Mais d'un autre côté, même si la mort temporelle concrète arrive de manière accidentelle, le fait de mourir est une vérité nécessaire sub specie aeternitatis, une vérité essentielle qui coexiste avec la durée d'un mode (SPE p. 292-293, SPP p. 58 $)^{11}$. Il semble alors que l'on reste avec, d'un côté, l'existence mortelle et finie, et de l'autre, les essences éternelles mais sans vie : la vie et la mort semblent entourées de toutes parts par la mort.

La réplique de Deleuze ferait référence à la réalité dynamique des essences, considérées comme des puissances pleinement effectives " d'exister et d'agir » (EPS p. 194, 304), ou comme des degrés de puissance d'agir et de penser (SPE p. 176, 282-283, 292). Une essence est aussi dynamique que le corps qui l'actualise, elle est de " l'énergie potentielle [...], l'énergie de l'événement pur », " tandis que les formes d'actualisation correspondent aux effectuations de l'événement " par l'entremise des parties extensives, ou corps ${ }^{12}$. La durée caractérise l'existence des modes dans la mesure où celle-ci consiste en " des variations continues de la puissance d'agir du mode existant " (SPP p. 87), et ce n'est qu'en ce sens que le temps mesure la puissance d'un mode : "les états de l'essence sont toujours aussi parfaits qu'ils peuvent l'être, mais diffèrent suivant leur loi de production dans l'existence ", selon que l'affect d'un mode reflète une augmentation ou une diminution de sa puissance d'agir (SPP p. 58). La qualité vécue de la durée consiste dans « les transitions vécues qui définissent les affects, passages constants à des perfections plus ou moins grandes » qu'on éprouve par la joie ou la tristesse (SPP p. 87, 57-58), affects qui « ne sont pas séparables de la durée qui les rattache à l'état précédent et les fait tendre à l'état suivant "(SPP p. 69 ; voir SPE p. 200).

De la sorte, « l'existence elle-même est [...] une sorte d'épreuve [...], une épreuve physique ou chimique, comme celles des artisans qui vérifient la qualité d'une matière" (SPE p. 296 ; voir SPP p. 58). La personne qui s'efforce de vivre autant que possible en accord avec son essence perdra peu en mourant ; elle perdra seulement les éléments passifs et conditionnés de

11. Voir aussi Deleuze, Différence et répétition, Paris, Presses Universitaires de France, 1968, p. 146-153 ; désormais $D R$.

12. Deleuze, Logique du sens, Paris, Éditions de Minuit, 1969, p. 125 ; désormais LS. 
l'extérieur de son corps et de son âme ; mais ce qui reste, le degré de puissance lui-même, "est absolument effectué » (SPE p. 293-298), à condition que pendant sa vie cette personne ait fait l'expérience du plus possible de joies actives, qui dépendent de notre seule puissance d'agir et de penser. De telles joies, qui expriment " la possession pleine, formelle » de la puissance ou perfection d'un mode, ne semblent avoir cours que dans le temps mesurable, mais elles sont en fait éternelles (SPP p. 58-59, 69-70) et nous permettent de rejoindre l'éternité durant notre vie (SPP p. 59-61). Ou, pour le dire autrement, selon le degré d'activité auquel nous parvenons, nous comprenons l'éternité de la vie elle-même comme une puissance d'agir et de penser ; non pas l'éternité qui persiste ou arrive après la mort, mais celle qui accompagne la vie et qui peut être « l'objet d'une expérience directe » (SPE p. 293 ; voir E V P23 Scolie). La mort, au contraire, est d'intensité nulle ; c'est le degré zéro de l'intensité ou de la puissance, et du processus de "devenir " d'une intensité à une autre ${ }^{13}$. Par conséquent, " toute intensité mène dans sa vie propre l'expérience de la mort, et l'enveloppe » (AO p. 395) plutôt que la mort ne limite la vie. Une essence ou un degré de puissance " forme toujours une limite (un maximum ou un minimum)» (SPE p. 186), non parce qu'il manquerait quoi que ce soit aux essences, mais parce que les modes existants qui leur correspondent peuvent s'approcher du maximum ou du minimum d'un degré de puissance donné, à un point tel que le mode réalise réellement sa puissance d'agir et de penser - et son conatus n'est, justement, que son effort pour faire cela (SPE p. 210).

La vie elle-même est alors éternelle quand elle est vécue avec une intensité maximale, ou quand l'existence extrinsèque et dans la durée d'un mode s'approche le plus près possible de la "force d'exister " (vis existendi), de l'élan vital de son essence. C'est pourquoi " un homme libre ne pense à rien moins qu'à la mort, et sa sagesse est une méditation non de la mort mais de la vie» (E IV P67).

\section{Durée et éternité}

Il devrait être évident par ce qui précède que la durée et l'éternité ne sont pas, chez Spinoza, opposées de la manière dont le croient les commentateurs. En fait, même Deleuze croit que Spinoza affirme " une distinction de nature entre la durée et l'éternité » (SPE p. 292). Toutefois, ceci n'est vrai que si l'on confond la durée avec le temps qui le mesure, et l'éternité avec l'éternité sans temps des vérités éternelles. Et aucune de ces deux suppositions n'est juste.

En elle-même, et indépendamment du temps qui la mesure, la durée est un devenir-actuel et une continuité dans l'actualité qui expriment la puissance active de Dieu en tant que natura naturans, par l'intermédiaire de la puissance

13. Deleuze, Gilles et Guattari, Félix, Capitalisme et schizophrénie: L'Anti-CEdipe, Paris, Éditions de Minuit, 1972, p. 393-395; désormais AO. 
d'agir qualitativement déterminée qui est un mode de l'essence éternelle. La durée d'un mode, ou son existence actuelle, ne suit pas du mode lui-même ou même de son essence, mais d'une cause efficiente extrinsèque qui a pour effet que les parties composantes du mode adoptent le rapport particulier de mouvement et de repos qui correspond à son essence ou à son degré de puissance ; et parce qu'il n'est pas causé par soi, il manque au mode l'être nécessaire qui appartient à la substance. Puisque l'éternité est « l'existence elle-même en tant qu'elle est conçue comme suivant nécessairement de la seule définition d'une chose éternelle " ( $E$ I Définition 8 ), on comprend pourquoi les modes ne sont pas éternels et pourquoi « bien que les modes existent, nous pouvons les concevoir comme n'existant pas [...] si nous avons égard à la seule essence des modes » (Lettre 12). Mais la durée, quoiqu'elle ne soit pas l'existence éternelle ou nécessaire, est une vis existendi, et non un écoulement déterminé de temps ; et, en fait, sa mesure propre n'est pas le temps mais l'essence qui mesure le degré selon lequel elle actualise sa puissance d'agir, l'essence établissant le maximum et le minimum de cette puissance. La durée découle des « choses éternelles ", c'est-à-dire des essences et des lois éternelles du mouvement et du repos ainsi que des lois de composition et de décomposition des corps. Quand on coupe la durée de sa source éternelle et qu'on la confond avec le temps, on se retrouve immédiatement au beau milieu des paradoxes de Zénon :

Dès que l'on aura conçu abstraitement la durée et que, la confondant avec le temps, on aura commencé de la diviser en parties, il deviendra impossible de comprendre en quelle manière une heure, par exemple, peut passer. Pour qu'elle passe, en effet, il sera nécessaire que la moitié passe d'abord, puis la moitié du reste et ensuite la moitié de ce nouveau reste, et retranchant ainsi à l'infini la moitié du reste, on ne pourra jamais arriver à la fin de l'heure (Lettre 12).

Ce passage aurait pu provenir de l'Évolution créatrice de Bergson ${ }^{14}$, et la solution de Spinoza aux paradoxes de Zénon, comme celle de Bergson, consiste à concevoir la durée comme n'ayant rien à voir avec les mesures temporelles, les «moments» dans le temps ou la chronologie ${ }^{15}$. La réalité de la durée est un devenir-actuel dans lequel le schéma passé-présent-futur qui est indissociable du temps n'a pas de place (voir $L S$ p. 9-18, 77-81, 176-177, 190-197, 206-207, 215-216). La durée est, selon l'expression de Bergson, " un devenir qui dure, un changement qui est la substance même » (B p. 29); c'est « le virtuel [...], en tant qu'il s'actualise, en train de s'actualiser " $(B \mathrm{p}$. 36), si l'on comprend le " virtuel » comme les essences de Spinoza qui, comme les « virtuels » de Bergson, sont " réels sans être actuels ».

Quant aux essences, quoique éternelles, elles ne sont pas " hors du temps » ou statiques à la manière des vérités logiques nécessaires. Les essen-

14. Bergson, Henri, L'évolution créatrice, Paris, Presses Universitaires de France, 1941, p. 303-313.

15. Voir Deleuze, Le Bergsonisme, Paris, Presses Universitaires de France (Quadrige), 1997 ; désormais $B$. 
ces, à la différence des modes existants finis, n'ont pas de durée, que celle-ci soit considérée en elle-même (comme vis existendi) ou à travers le temps. Les essences sont des degrés de puissance qui correspondent à un certain rapport de mouvement et de repos entre les « corps simples » infiniment petits qui constituent un individu existant, mais elles ne sont pas identiques au mouvement et au repos de ces corps. Pourtant, bien que le mouvement et le repos actuels des corps ne constituent pas d'essence, ils en expriment concrètement une, et cette essence-là " contient » implicitement alors le mouvement et le repos, étant donné qu'elle détermine nécessairement un rapport de mouvement et de repos. De plus, le mouvement et le repos des corps sont des notions comparatives, des manières de distinguer les corps les plus simples entre eux (E II P13 Lemme 1), et par conséquent ils impliquent le temps, ou la mesure de la durée par la comparaison des actions d'un corps avec les actions d'un autre : la vitesse et le repos d'un corps sont relatifs à d'autres corps. En ce sens, les essences éternelles contiennent implicitement à la fois la durée, et le temps, quoique les essences ne soient pas dans le temps. En somme, les essences ne sont pas statiques mais dynamiques, et elles renvoient nécessairement au temps (taux de changement, vitesse et lenteur). La sorte de "nécessité éternelle » qui se trouve dans les essences (SPE p. 291 ; E V P22 Démonstration) appartient aux rapports de mouvement et de repos qui enveloppent nécessairement le temps. Il y a, alors, du temps dans l'éternité, sous la forme des rapports de mouvement et de repos des corps - mais conçus sub specie aeternitatis, par une appréhension de la nécessité par la raison, plutôt que saisis dans l'expérience ordinaire dans le temps par l'imagination.

$\mathrm{Si}$, en un sens, il y a du temps dans l'éternité, il est tout aussi vrai qu'il y a de l'éternité dans le temps, ou du moins dans la durée. Chaque mode fini, lorsqu'il existe, exprime une essence ou un degré de puissance particulier qui fixe les limites minimales et maximales de sa puissance d'exister. Un mode est d'autant plus actif, et il s'approche d'autant plus de sa puissance d'exister, que ses actions suivent de sa propre essence ou de sa propre puissance d'action. De plus, un mode est d'autant plus libre que ses actions sont déterminées par sa propre nature ou suivent nécessairement d'elle (E I Définition 7) et qu'il s'approche du statut de cause de soi (E I Définition 1). Un mode, par conséquent, participe d'autant plus de l'éternité de l'existence d'un être cause de soi qu'il maximise sa puissance et se détermine lui-même.

On peut faire l'expérience de l'éternité dans le temps, par les affects actifs et l'autodétermination rationnelle, parce que l'éternité elle-même, quoiqu'elle ne soit ni dans le temps ni équivalente à une durée infinie, existe à travers le temps, sous-tendant les événements qui se déploient dans le temps chronologique, en tant que nécessité rationnelle qui explique cette succession apparemment contingente. C'est pourquoi «dans l'éternité il n'y a [...] ni quand, ni avant, ni après » (E I P33 Scolie 2). Mais la nécessité impliquée dans l'existence éternelle est celle de l'autodétermination, les actions et affections d'une chose suivant seulement de la nécessité de sa propre nature — ce n'est pas celle d'une 
éternité «abstraite », statique et "hors du temps» au sens platonicien du terme. En bref, l'éternité est la puissance de Dieu lui-même, l'existence éternelle et l'activité éternelle, et pas un ensemble de relations logiques ou de vérités a priori abstraites : la nécessité éternelle est existentielle et non pas simplement conceptuelle. Elle est, certes, en dehors de l'écoulement chronologique du temps, mais elle n'est pas totalement atemporelle, et alors qu'il est vrai que les relations de cause à effet ne sont correctement comprises qu'à travers leur relation atemporelle de condition nécessaire et suffisante à conséquent, cette nécessité rationnelle sous-tend mais ne remplace pas l'efficacité causale de la puissance productrice divine. Pour être exercée, cette puissance conditionnante auto-conditionnée doit se différencier en cause et effet, en raison et conséquence; ce qu'elle fait par les différences qualitatives entre les essences modales, différences qu'elle produit activement simplement en les affirmant, et en affirmant par là à la fois les différences entre les essences et la différence de chaque essence (voir DR p. 119-128, 152-153, 163-165). En ce sens, l'éternité se redouble, ou elle "retourne " à elle-même. Elle se "précède " elle-même métaphysiquement, mais pas dans le temps chronologique. Toutefois, cette "préséance » est le genre de temps qui appartient à l'éternité elle-même, à savoir, à l'existence éternelle en tant que nécessité se déterminant elle-même.

Il est vrai que c'est là une conception très deleuzienne de Spinoza, et par là une conception bergsonienne, une conception nietzschéenne. Il se pourrait malgré tout qu'elle soit vraie. À défaut d'autre chose, nous espérons au moins que notre lecture est suffisamment fondée dans l'Éthique de Spinoza pour rendre questionnables quelques unes des idées établies concernant la durée, le temps et l'éternité dans la philosophie de Spinoza, et pour montrer qu'une philosophie spinoziste du temps n'est pas l'absurdité qu'elle peut sembler au premier abord ${ }^{16}$.

\section{Bibliographie :}

Bennett, Jonathan : A Study of Spinoza's Ethics, Indianapolis, Hackett, 1984. Bergson, Henri : L'évolution créatrice, Paris, Presses Universitaires de France, 1941.

Deleuze, Gilles : Le Bergsonisme, Paris, Presses Universitaires de France (Quadrige), 1997.

- Différence et répétition, Paris, Presses Universitaires de France, 1968.

- Logique du sens, Paris, Éditions de Minuit, 1969.

- Spinoza et le problème de l'expression, Paris, Éditions de Minuit, 1968.

- Spinoza : Philosophie pratique, Paris, Éditions de Minuit, 1981.

16. Ce texte a été traduit de l'anglais par Syliane Charles. 
Deleuze, Gilles et Guattari, Félix : Capitalisme et schizophrénie : L'AntiEdipe, Paris, Éditions de Minuit, 1972.

Hampshire, Stuart : Spinoza, Harmondsworth, Penguin, 1978,

Spinoza, Benedictus : Éthique, dans Ch. Appuhn, Euvres, Paris, GF, 1965, vol. III.

- Lettres, dans Ch. Appuhn, Euvres, Paris, GF, 1966, vol. IV.

Wild, John, éd. : Spinoza : Selections, New York, Charles Scribner's Sons, 1958. 